\title{
Rigidez en cirugía de reconstrucción del ligamento cruzado anterior
}

\section{Knee Stiffness in Anterior Cruciate Ligament Reconstruction}

\author{
David Figueroa $^{1}$ Jaime Espinoza ${ }^{2}$ Claudio Yañez ${ }^{2}$ \\ ${ }^{1}$ Departmento de Traumatología y Ortopedia, Clínica Alemana de \\ Santiago SA, Vitacura, Región Metropolitana, Chile \\ ${ }^{2}$ Clínica Alemana Santiago, Universidad del Desarrollo, Vitacura, \\ Región Metropolitana, Chile
}

Rev Chil Ortop Traumatol 2018;59:95-99.

\author{
Nazira Bernal²
}

\begin{abstract}
Address for correspondence Nazira Bernal, MD, Departamento de Traumatología y Ortopedia, Clínica Alemana de Santiago SA, Vitacura, Región Metropolitana, Chile (e-mail: Nazirabbader@gmail.com).
\end{abstract}

\section{Resumen \\ Palabras-clave \\ - artrofibrosis \\ - rigidez de rodilla \\ - artrofibrolisis \\ - cirugia ligamentaria}

La rigidez de rodilla en cirugía de Reconstrucción del Ligamento Cruzado Anterior (R-LCA) es una complicación devastadora, alcanzando una incidencia variable entre el $4 \%$ y el $38 \%$. La definición de artrofibrosis es aún poco clara, lo que ha llevado a distintos esquemas terapéuticos. Conocer la etiopatogenia es clave para entender los procesos y las posibles alternativas de tratamiento. Distintas clasificaciones han sido descritas, siendo la de Shelbourne la más usada, debido a su valor pronóstico asociado. El objetivo del tratamiento en una rodilla estable es mejorar la movilidad articular, la satisfacción del paciente, y disminuir el riesgo de artrosis a largo plazo. El tratamiento se puede dividir en quirúrgico y conservador. Ese último, se enfoca principalmente en buscar la causa y lograr una prevención e intervención temprana, siendo el manejo que con mayor frecuencia se realiza. El tratamiento quirúrgico es una opción cuando el tratamiento conservador falla. Se realizó una revisión de la literatura y de 150 pacientes sometidos a R-LCA, de los cuales 4 presentaron artrofibrosis a un seguimiento de 2 años. Además, presentamos nuestro algoritmo de manejo terapéutico.

Knee stiffness in Anterior Cruciate Ligament Reconstruction (ACL-R) is a devastating complication, with a variable incidence of $4 \%$ to $38 \%$. The definition of arthrofibrosis is still unclear, which has led to different therapeutic schemes. Knowing the etiopathogenesis is key to understanding the processes and possible treatment alternatives. Different classifications have been described, with Shelbourne being the most used, due to its associated prognostic value. The aim of treatment in a stable knee is to improve joint mobility, patient satisfaction, and decrease the risk of long-term osteoarthritis. The treatment can be divided into operative and non-operative. The latter focuses mainly on finding the cause and achieving prevention and early intervention, being the management that is most frequently performed. Surgical treatment is an option when conservative treatment fails. A review of the literature and of 150 patients undergoing R-LCA was performed, of which 4 presented arthrofibrosis at a 2-year follow-up. In addition, we present our therapeutic management algorithm. received

October 28, 2018

accepted

December 4, 2018
DOI https://doi.org/

10.1055/s-0039-1677756. ISSN $0716-4548$.
Copyright $\odot 2018$ by Thieme Revinter Publicações Ltda, Rio de Janeiro, Brazil
License terms

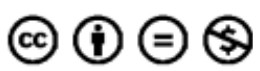




\section{Introducción}

La rigidez de rodilla o artrofibrosis en cirugía de reconstrucción del ligamento cruzado anterior (R-LCA) de la rodilla es una complicación devastadora, que varía según la literatura con una incidencia reportada de un $4 \%$ a un $38 \% .^{1-3}$ De las R-LCA, el $23 \%$ están asociadas a reparación del ligamento colateral medial (LCM) de rodilla ipsilateral. ${ }^{4}$ En lesiones traumáticas, como en las luxaciones de rodilla, se ha asociado entre un $30 \%$ a un $50 \%$ de rigidez, requiriendo algún procedimiento adicional en el futuro. ${ }^{5,6}$ En el tratamiento conservador, el enfoque principal es buscar la causa de la rigidez, pero es fundamental lograr una prevención e intervención tempranas.

\section{Etiopatogenia}

La organización y homeostasis de los tejidos, depende de constantes señales de citoquinas y factores de crecimiento activados localmente. Esas citoquinas coordinan tanto el crecimiento, diferenciación y apoptosis celular, mediante señales entre células locales (paracrino), como también señales de la misma célula (autocrina). ${ }^{7}$

Factores de crecimiento liberados por plaquetas como TGF-B, juegan un rol central en los procesos de reparación de los tejidos. ${ }^{8}$ En el momento de una injuria del tejido, ellos inician una cascada de eventos, resultando en la producción de proteínas de matriz extracelular e inhibidores de proteasas y enzimas proteolíticas. A la formación de matriz extracelular se agrega además colágeno, fibronectina y proteoglicanos. Un incremento local de TGF-B, desencadena un feedback negativo, logrando un equilibrio. La sobreexpresión de TGF-B, resultará en un depósito progresivo de matriz extracelular y tejido fibroso. ${ }^{9,10}$ Esa sobre expresión puede resultar a largo plazo en el resultado de artrosis secundaria, como una de las teorías expuestas.

En 1987, Paulos y cols.," describe el "síndrome de contractura infrapatelar" (IPCS), ubicado en el intervalo anterior o compartimiento anterior, espacio limitado entre el tendón patelar, la grasa infrapatelar anterior, ligamento intermeniscal transverso $\mathrm{y}$ tibia por posterior. Los responsables de ese fenómeno de fibrosis serían el trauma o hemorragia articular, que resulta en una fibrosis hiperplásica del compartimiento anterior. También se ha asociado a inmovilización prolongada y un déficit de extensión. El IPCS es una causa infrecuente de morbilidad post-traumática de la rodilla, en particular en reconstrucción de ligamento cruzado anterior. Se caracteriza por una restricción de la extensión y flexión de la rodilla, asociado a una rigidez de la excursión normal de la patela. ${ }^{11}$ Se estratificaron los pacientes con IPCS dentro de 3 estados: Prodromal, activo y residual. En el estado prodromal se caracteriza por inflamación y edema peri articular, debilidad y retraso en la activación de cuádriceps, déficit de extensión, ROM doloroso y sensibilidad sobre el tendón patelar. En el estado activo, se presenta una dramática pérdida de movilidad de la patela, marcada atrofia de cuádriceps, perdida de ROM e induración del tejido graso, asociado a rigidez del tendón patelar. En el estado residual, predomina el tejido peripatelar y retinacular más flexible que en el estado activo, atrofia marcada de cuádriceps y perdida de flexión y extensión. La relevancia de ese cuadro en el estado residual es la progresión de la artrosis patelo-femoral y patela ínfera.

\section{Definición}

La definición de artrofibrosis varía ampliamente, siendo incierto el tratamiento. Shelbourne y cols., la define como una pérdida de extensión de menos de 15 grados. ${ }^{12}$ Mayr y cols., lo define como una pérdida del rango de movilidad, asociado a tejido cicatricial en algún compartimiento. ${ }^{13}$ Clínicamente, se caracteriza como una limitación del rango de movilidad sintomática en rodillas intervenidas quirúrgicamente. ${ }^{12,14}$

\section{Clasificación}

Se han propuesto una variedad de tipos de clasificaciones. Sprague y cols., define la pérdida de movilidad en base a la distribución anatomo-patologica. ${ }^{15}$ Pizzo y cols., graduó la pérdida de ROM, evaluando desde la flexión y extensión completa, con severa pérdida de movilidad considerada mayor a 10 grados desde extensión completa y menor a 90 grados de flexión. ${ }^{16}$ Blauth y Jaeger, graduaron la pérdida de movilidad basado sobre el arco de movilidad completa, como grado I (leve): ROM mayor 120 grados de flexión, Grado II (moderado): ROM 80-120 grados, grado III (severo) ROM 40 a 80 grados y grado IV (extremo): menor a 40 grados de flexión. ${ }^{17}$ La más reciente clasificación descrita por Shelbourne y cols., ${ }^{18}$ compara la pérdida de movilidad con la rodilla contralateral del paciente (Tabla $\mathbf{1}$ ).

\section{Etiología}

Las causas de rigidez en cirugía ligamentaria de rodilla, son variadas. A continuación, se describen acotadamente cada una de ellas.

- Errores técnicos: Se ha demostrado que la adecuada posición del injerto es esencial para reducir la pérdida de movilidad. ${ }^{19}$ (Tabla 2)

- Tensión del injerto: Se sugiere el incremento de la tensión del injerto, resultando en una pérdida de movilidad secundaria a una sobre constricción de la articulación, aunque esa relación sigue siendo controversial. Markolf y cols., en un estudio biomecánico demostró que sobre tensionar el injerto conducía a laxitud anteroposterior, inestabilidad, pobre integración del injerto y falla de la plastía. ${ }^{20,21}$

Tabla 1 Clasificación de Shelbourne y cols.

\begin{tabular}{|l|l|l|}
\hline Tipo & Flexión & Extensión \\
\hline 1 & Normal & $<10$ \\
\hline 2 & Normal & $>10$ \\
\hline 3 & $>25$ & $>19$ \\
\hline 4 & $>30$ & $>10$ con patela ínfera. \\
\hline
\end{tabular}


Tabla 2 Grupo de pacientes tratados en nuestro centro

\begin{tabular}{|l|l|l|l|l|l|l|l|l|l|}
\hline Nombre & Edad & Sexo & $\begin{array}{l}\text { Tiempo } \\
\text { R-LCA }\end{array}$ & Injerto & $\begin{array}{l}\text { ROM } \\
\text { pre-AA }\end{array}$ & $\begin{array}{l}\text { ROM } \\
\text { actual }\end{array}$ & Proced. & $\begin{array}{l}\text { Lesiones } \\
\text { asociadas }\end{array}$ & $\begin{array}{l}\text { Tiempo de procedimiento } \\
\text { (R-LCA-AA) }\end{array}$ \\
\hline F.L.G. & $18 \mathrm{a}$ & $\mathrm{F}$ & 21 días & ST-G & $-5-0-100$ & $0-130$ & AA & LMM (S) & 8 meses \\
\hline T.S.A. & $21 \mathrm{a}$ & $\mathrm{M}$ & 13 días & ST-G & $-5-100$ & $0-120$ & AA & no & 6 meses \\
\hline G.B.B. & $40 \mathrm{a}$ & $\mathrm{F}$ & 60 días & ST-G & $-10-0-105$ & $0-125$ & AA & no & 6 meses \\
\hline F.S.L. & 18 & F & 22 días & HTH & $-5-85$ & $0-125$ & AA & no & 6 meses \\
\hline
\end{tabular}

Abreviaciones: AA, artrolisis artroscópica; Proced., procedimiento; R-LCA, reconstrucción del ligamento cruzado anterior.

- Elección del injerto: La relación es cuestionada. Pinczewski y cols., demostró en un estudio prospectivo que el 31\% HTH y $19 \%$ ST-G tenían déficit de extensión con un seguimiento a 5 años, siendo no estadísticamente significativo. ${ }^{22}$ Por otra parte, Sajovic y cols., demostró que no habían diferencias significativas a 5 años de seguimiento. ${ }^{23}$

- Procedimientos extrarticulares y lesiones a tejidos blandos: Se ha asociado la reparación del LCM en conjunto a una R-LCA con pérdida de movilidad. ${ }^{24}$ Cosgarea y cols., ${ }^{25}$ encontró que procedimientos asociados a R-LCA como reparación meniscal y meniscectomía no incrementan el riesgo de artrofibrosis. Por otro lado, el trauma a tejidos blandos aumenta el riesgo.

- Tiempo de la cirugía: El momento adecuado de cuando realizar la cirugía sigue siendo controversial. Shelborne y cols., ${ }^{26}$ evaluó 129 atletas con el diagnostico de rotura de LCA. El grupo en el cual se realizó la R-LCA después de las 3 semanas, se obtuvo una incidencia menor de artrofibrosis, siendo estadísticamente significativo $(p<0,05)$. Wasilewski y cols., ${ }^{27}$ demostró que el $22 \%$ presentaban rigidez cuando la reconstrucción de LCA se realizaba antes de las 4 semanas.

- Inmovilización: La inmovilización post cirugía ligamentaria, tiene una aceptación como factor de riesgo. Hooper y Walton, reportaron que el $46 \%$ de los pacientes con inmovilización de 2 semanas, tienen algún grado de pérdida de movilidad. ${ }^{28}$

- Genética: Algunos pacientes evolucionan con artrofibrosis a pesar de que se tomen todas las precauciones, por lo que se plantea si hay alguna predisposición genética. Skutek, ${ }^{1}$ evaluó 17 pacientes con artrofibrosis después de la R-LCA, tomando muestras de $A D N$ en sangre, demostrando que paciente con artrofibrosis presentan alelo HLA-Cw*08, por lo que se infiere que ese sería un responsable. Se desconoce si esos alelos representan un aumento potencial del riesgo de artrofibrosis, ya sea por una mayor susceptibilidad o una disminución de los mecanismos de defensa.

\section{¿Qué nos dice la Evidencia?}

Sanders y cols, ${ }^{29}$ en una cohorte demostró que el riesgo de artrofibrosis es 2,5 veces mayor en mujeres, el cual se asemeja a la literatura. Además, demostró que pacientes intervenidos mayor a 4 semanas tienen un significativo mayor riesgo de artrofibrosis que los pacientes tratados entre las 4 semanas. La inmovilización prolongada sigue siendo un factor de riesgo a considerar. La reparación meniscal concomitante demostró mayor riesgo de pérdida de movilidad, en comparación con pacientes sin reparación. La elección del injerto, al comparar HTH versus ST-G, ese último está asociado a un mayor riesgo (HTH 0,3 v/s ST-G 6,8).

En cuanto al tiempo desde el evento hasta la cirugía mencionado anteriormente, muchos trabajos muestran un significativo riesgo al realizar una intervención en atletas antes de la primera semana post trauma. Hoy en día, se propone el uso del concepto del estado inflamatorio de la rodilla como factor más importante para definir el momento adecuado para la R-LCA. ${ }^{30}$

\section{Tratamiento}

Hay un consenso generalizado que la prevención es la principal medida. El tratamiento se puede dividir en conservador y quirúrgico. En el primero, se enmarca dentro del tratamiento profilaxico, en el que se incluyen el uso de AINES, rehabilitación temprana, ejercicios con movilizador continuo pasivo (MPC), uso de férulas y braces, corticoesteroides y manipulación bajo anestesia. El tratamiento quirúrgico es de elección para paciente con un déficit de flexión y sin déficit de extensión. La mayoría de los estudios muestra ganancia solo parcial del rango en flexión y no está excepta de otras complicaciones como fracturas, daño condral, y lesión de la plastía ligamentosa. Se ha demostrado un beneficio, cuando se asocia a procedimientos artroscópicos. En la actualidad, no hay un consenso claro para elegir el procedimiento ideal en relación al tipo de paciente, ROM y tiempo post operatorio. Dentro de las alternativas, se dispone principalmente de movilización más artrolisis artroscópica, técnicas abiertas y cirugías de salvataje. ${ }^{31}$ La artrolisis artroscópica (AA), es el procedimiento más usado. ${ }^{32,33}$ Hermann y cols. ${ }^{34}$ en el año 2017, publicó resultados a largo plazo (17 años) de la AA después de una reconstrucción de ligamento cruzado anterior. El ROM se encontró entre mayor de 5 grados de déficit de extensión y mayor de 15 grados de déficit de flexión. Hubo una correlación significativa de artrofibrosis en menores de 4 semanas desde el evento traumático $(p<.0001)$. La mayoría de las AA se realizó entre el primer y segundo año post operado (42,6\%). La lesión asociada más frecuente es el menisco medial con un $32 \%$, con IKDC con 89,9 con menisco intacto o suturado versus 81,6 con meniscectomía, pero sin significancia estadística (p 0.039). 

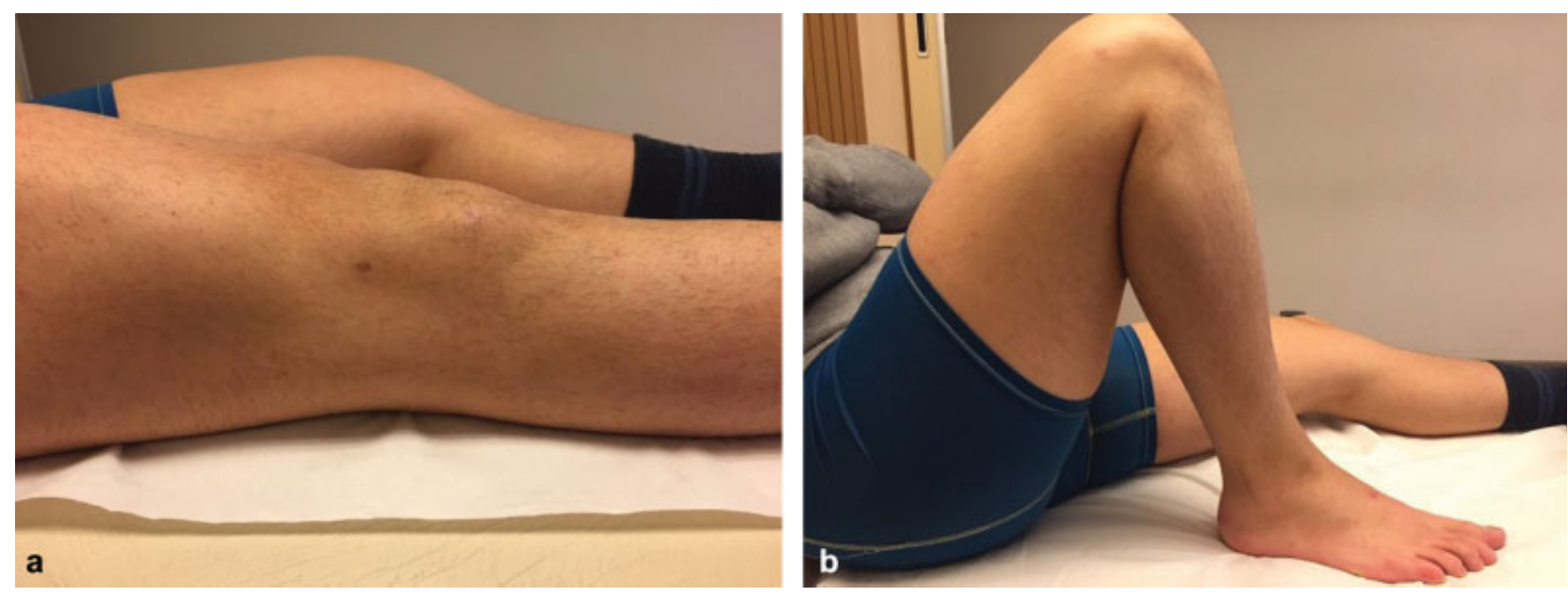

Fig. 1 Paciente con dos años de post operatorio de reconstrucción de ligamento cruzado anterior, evolucionando con déficit del rango de movilidad. Resultados post AA (a) extensión y (b) flexión.

No hay evidencia suficiente para la elección de un injerto sobre el otro. ROM puede mejorar con artrolisis artroscópica a largo plazo. La pérdida de movilidad articular se ha asociado a un alto grado de desarrollar artrosis y los peores resultados fueron en una intervención realizada mayor a 1 año del post operatorio.

Es importante destacar que el tratamiento con mejores resultados en la actualidad, es la prevención y una temprana intervención. ${ }^{33}$ Noyes y cols., en un estudio de 203 pacientes con reconstrucción R-LCA con aloinjerto, seguido inmediatamente por un programa de rehabilitación y una intervención programada de la limitación de rango de movilidad, el $98 \%$ logran un rango de movilidad completo. ${ }^{35}$ En la mayoría de los casos se puede prevenir, retrasando la reconstrucción del LCA hasta que el paciente tenga un derrame mínimo o nulo en la rodilla lesionada, un buen rango de movimiento (preferiblemente más de 120 grados), un buen control del cuádriceps y una marcha normal.

En una revisión sistemática del 2017, que compara opciones quirúrgicas abiertas y artroscópicas, Ekhtiari y cols., demostró mejores resultados en cuanto al ROM en flexión y extensión. La movilización bajo anestesia logra una flexión post operatoria de un 47,8 grado, en cambio, el uso de braces y férulas, mostró una mayor extensión con 6,2 grados. ${ }^{36}$

\section{Nuestros Enfrentamientos}

Se realizó una revisión retrospectiva de 150 pacientes entre 13 a 40 años sometidos a R-LCA con un seguimiento promedio de 2 años. De ellos, 4 pacientes presentaron artrofibrosis. El $75 \%$ de ellos fueron mujeres y el injerto más usado fue el de isquiotibiales con un $75 \%$. El ROM preoperatorio de los pacientes con artrofibrosis en promedio fue de $-10^{\circ}$ de extensión y $105^{\circ}$ de flexión. Mejorando a un ROM post tratamiento de $0^{\circ}$ de extensión y $125^{\circ}$ de flexión. Todos fueron sometidos a artrolisis artroscópica, 6 meses post R-LCA en promedio, luego de la falla del manejo conservador. En síntesis, de 150 R-LCA realizadas en los últimos 2 años en nuestro departamento, un 2,6\% evolucionaron con limitación del rango de movilidad, que requirieron un tratamiento quirúrgico, logrando excelentes resultados con la artrolisis artroscópica (-Fig. 1).

\section{Conclusión}

La artrofibrosis posterior a una R-LCA es una complicación que, aunque es poco frecuente, es devastadora. Existe una definición poco clara e inadecuada, lo que dificulta el algoritmo de manejo, siendo la prevención y la intervención temprana las medidas más importantes. Nuestro enfrentamiento consiste en un algoritmo agresivo, realizando una artrolisis artroscópica en los primeros 6 meses posterior a la R-LCA con buenos resultados postoperatorios.

\section{Conflicto de Interés}

Dr. Jaime Espinoza Valdés: No presenta conflictos de interés.

Dr. David Figueroa Poblete: Editor de Revista de la Sociedad Chilena de Ortopedia y Traumatología.

Dr. Claudio Yáñez Lagos: No presenta conflictos de interés. Dra. Nazira Bernal Bader: No presenta conflictos de interés.

\section{Bibliografía}

1 Krenn V, Ruppert M, Knöß P, et al. [Synovialitis of the arthrofibrotic type: criteria of a new synovialitis type for the diagnosis of arthrofibrosis]. Z Rheumatol 2013;72(03):270-278. Doi: 10.1007/s00393-012-1076-7

2 Kartus J, Magnusson L, Stener S, Brandsson S, Eriksson BI, Karlsson J. Complications following arthroscopic anterior cruciate ligament reconstruction. A 2-5-year follow-up of 604 patients with special emphasis on anterior knee pain. Knee Surg Sports Traumatol Arthrosc 1999;7(01):2-8. Doi: 10.1007/s001670050112

3 Langer F, Gross AE, Greaves MF. The auto-immunogenicity of articular cartilage. Clin Exp Immunol 1972;12(01):31-37

4 Noyes FR, Mangine RE, Barber SD. The early treatment of motion complications after reconstruction of the anterior cruciate ligament. Clin Orthop Relat Res 1992;(277):217-228

5 Sisto DJ, Warren RF. Complete knee dislocation. A follow-up study of operative treatment. Clin Orthop Relat Res 1985;(198):94-101 
6 Shapiro MS, Freedman EL. Allograft reconstruction of the anterior and posterior cruciate ligaments after traumatic knee dislocation. Am J Sports Med 1995;23(05):580-587

7 Magit D, Wolff A, Sutton K, Medvecky MJ. Arthrofibrosis of the knee. J Am Acad Orthop Surg 2007;15(11):682-694

8 Border WA, Noble NA. Transforming growth factor beta in tissue fibrosis. N Engl J Med 1994;331(19):1286-1292

9 Bonner JC. Regulation of PDGF and its receptors in fibrotic diseases. Cytokine Growth Factor Rev 2004;15(04):255-273

10 Gharaee-Kermani M, Phan SH. Role of cytokines and cytokine therapy in wound healing and fibrotic diseases. Curr Pharm Des 2001;7(11):1083-1103

11 Paulos LE, Rosenberg TD, Drawbert J, Manning J, Abbott P. Infrapatellar contracture syndrome. An unrecognized cause of knee stiffness with patella entrapment and patella infera. Am J Sports Med 1987;15(04):331-341

12 Shelbourne KD, Wilckens JH, Mollabashy A, DeCarlo M. Arthrofibrosis in acute anterior cruciate ligament reconstruction. The effect of timing of reconstruction and rehabilitation. Am J Sports Med 1991;19(04):332-336

13 Mayr HO, Weig TG, Plitz W. Arthrofibrosis following ACL reconstruction-reasons and outcome. Arch Orthop Trauma Surg 2004;124(08):518-522

14 Magit D, Wolff A, Sutton K, Medvecky MJ. Arthrofibrosis of the knee. J Am Acad Orthop Surg 2007;15(11):682-694

15 Sprague NF III, O'Connor RL, Fox JM. Arthroscopic treatment of postoperative knee fibroarthrosis. Clin Orthop Relat Res 1982; (166):165-172

16 Del Pizzo W, Fox JM, Friedman ML, et al. Operative arthroscopy for the treatment of arthrofibrosis of the knee. Contemp Orthop 1985; 10:67-72

17 Blauth W, Jaeger T. [Arthrolysis of the knee joint]. [German] Orthopade 1990;19(06):388-399

18 Shelbourne KD, Patel DV, Martini DJ. Classification and management of arthrofibrosis of the knee after anterior cruciate ligament reconstruction. Am J Sports Med 1996;24(06):857-862

19 Yaru NC, Daniel DM, Penner D. The effect of tibial attachment site on graft impingement in an anterior cruciate ligament reconstruction. Am J Sports Med 1992;20(02):217-220

20 Bach BR Jr, Jones GT, Sweet FA, Hager CA. Arthroscopy-assisted anterior cruciate ligament reconstruction using patellar tendon substitution. Two- to four-year follow-up results. Am J Sports Med 1994;22(06):758-767

21 Markolf KL, Burchfield DM, Shapiro MM, Davis BR, Finerman GA, Slauterbeck JL. Biomechanical consequences of replacement of the anterior cruciate ligament with a patellar ligament allograft. Part I: insertion of the graft and anterior-posterior testing. J Bone Joint Surg Am 1996;78(11):1720-1727

22 Pinczewski LA, Deehan DJ, Salmon LJ, Russell VJ, Clingeleffer A. A five-year comparison of patellar tendon versus four-strand hamstring tendon autograft for arthroscopic reconstruction of the anterior cruciate ligament. Am J Sports Med 2002;30(04):523-536

23 Sajovic M, Vengust V, Komadina R, Tavcar R, Skaza K. A prospective, randomized comparison of semitendinosus and gracilis tendon versus patellar tendon autografts for anterior cruciate ligament reconstruction: five-year follow-up. Am J Sports Med 2006;34(12): 1933-1940

24 Harner CD, Irrgang JJ, Paul J, Dearwater S, Fu FH. Loss of motion after anterior cruciate ligament reconstruction. Am J Sports Med 1992;20(05):499-506

25 Dodds JA, Keene JS, Graf BK, Lange RH. Results of knee manipulations after anterior cruciate ligament reconstructions. Am J Sports Med 1991;19(03):283-287

26 Shelbourne KD, Wilckens JH, Mollabashy A, DeCarlo M. Arthrofibrosis in acute anterior cruciate ligament reconstruction. The effect of timing of reconstruction and rehabilitation. Am J Sports Med 1991;19(04):332-336

27 Wasilewski SA, Covall DJ, Cohen S. Effect of surgical timing on recovery and associated injuries after anterior cruciate ligament reconstruction. Am J Sports Med 1993;21(03):338-342

28 Hooper GJ, Walton DI. Reconstruction of the anterior cruciate ligament using the bone-block iliotibial-tract transfer. J Bone Joint Surg Am 1987;69(08):1150-1154

29 Sanders TL, Kremers HM, Bryan AJ, Kremers WK, Stuart MJ, Krych AJ. Procedural intervention for arthrofibrosis after ACL reconstruction: trends over two decades. Knee Surg Sports Traumatol Arthrosc 2017;25(02):532-537

30 Kwok CS, Harrison T, Servant C. The optimal timing for anterior cruciate ligament reconstruction with respect to the risk of postoperative stiffness. Arthroscopy 2013;29(03):556-565

31 Magit D, Wolff A, Sutton K, Medvecky MJ. Arthrofibrosis of the Knee. J Am Acad Orthop Surg 2007;15(11):682-694

32 Millett PJ, Wickiewicz TL, Warren RF. Motion loss after ligament injuries to the knee. Part II: prevention and treatment. Am J Sports Med 2001;29(06):822-828

33 Chen MR, Dragoo JL. Arthroscopic releases for arthrofibrosis of the knee. J Am Acad Orthop Surg 2011;19(11):709-716

34 Mayr HO, Brandt CM, Weig T, et al. Long-term Results of Arthroscopic Arthrolysis for Arthrofibrosis After Anterior Cruciate Ligament Reconstruction. Arthroscopy 2017;33(02): 408-414

35 Noyes FR, Berrios-Torres S, Barber-Westin SD, Heckmann TP. Prevention of permanent arthrofibrosis after anterior cruciate ligament reconstruction alone or combined with associated procedures: a prospective study in 443 knees. Knee Surg Sports Traumatol Arthrosc 2000;8(04):196-206

36 Ekhtiari S, Horner NS, de Sa D, et al. Arthrofibrosis after ACL reconstruction is best treated in a step-wise approach with early recognition and intervention: a systematic review. Knee Surg Sports Traumatol Arthrosc 2017;25(12):3929-3937 